University of South Florida

DIGITAL COMMONS

Digital Commons @ University of

@ UNIVERSITY OF SOUTH FLORIDA

South Florida

\title{
Smartphone-based Connected Bicycle Prototype Development for Sustainable Multimodal Transportation System
}

CUTR

Follow this and additional works at: https://digitalcommons.usf.edu/cutr_nctr

\section{Recommended Citation}

"Smartphone-based Connected Bicycle Prototype Development for Sustainable Multimodal Transportation System," National Center for Transit Research (NCTR) Report No. CUTR-NCTR-RR-2018-03, Center for Urban Transportation Research, University of South Florida, 2018.

DOI: https://doi.org/10.5038/CUTR-NCTR-RR-2018-03

Available at: https://scholarcommons.usf.edu/cutr_nctr/7

This Technical Report is brought to you for free and open access by the National Center for Transit Research (NCTR) Archive (2000-2020) at Digital Commons @ University of South Florida. It has been accepted for inclusion in Research Reports by an authorized administrator of Digital Commons @ University of South Florida. For more information, please contact digitalcommons@usf.edu. 
Final Report

\title{
Smartphone-based Connected Bicycle Prototype Development for Sustainable Multimodal Transportation System
}

\author{
Project No. \\ USDOT DTRT13-G-UTC56 Modification No. 3 \\ USF 21179063-17 \\ Prepared For \\ National Center for Transit Research
}

August 2019

\section{USF UNIVERSITY OF




\section{Final Report}

\section{USDOT DTRT13-G-UTC56 Modification No. 3}

\section{USF 21179063-17}

\section{Prepared for:}

\section{National Center for Transit Research}

\section{Prepared by:}

\section{USF Center for Urban Transportation Research}

Achilleas Kourtellis, Ph.D.

Pei-Sung Lin, Ph.D., P.E., PTOE

Neha Kharkar

August 2019 


\section{Disclaimer}

The contents of this report reflect the views of the authors, who are responsible for the facts and the accuracy of the information presented herein. This document is disseminated under the sponsorship of the U.S. Department of Transportation's University Transportation Centers Program, in the interest of information exchange. The U.S. Government assumes no liability for the contents or use thereof. 


\section{TABLE OF CONTENTS}

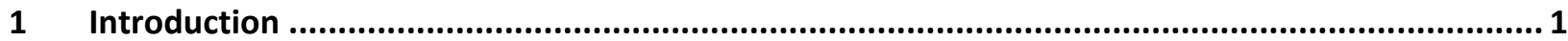

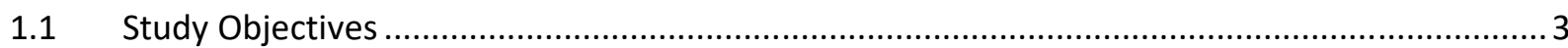

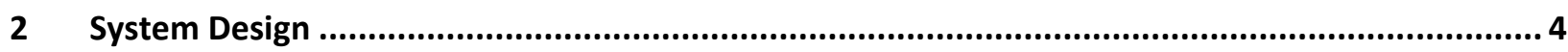

2.1 Identification and Selection of Parameters ............................................................... 4

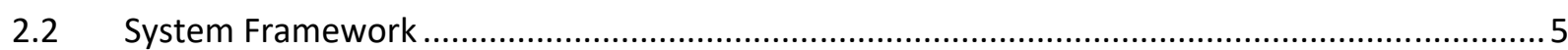

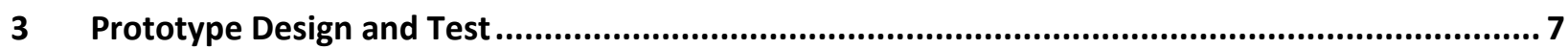

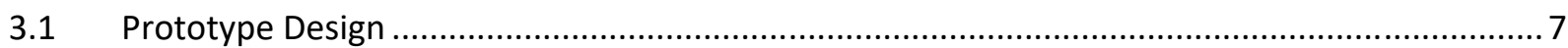

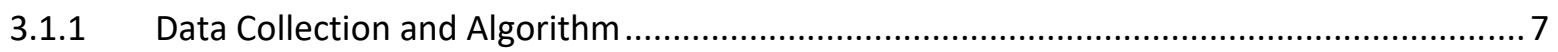

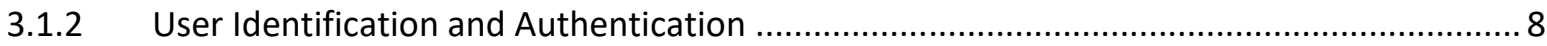

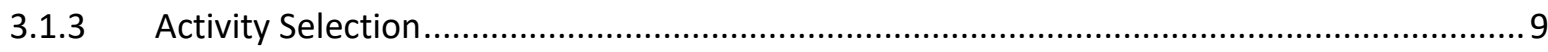

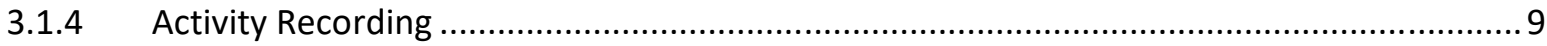

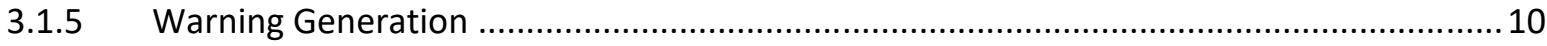

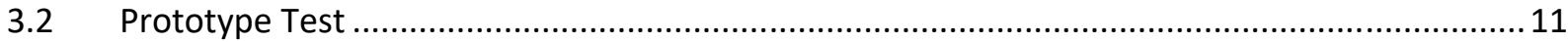

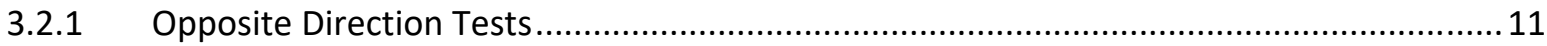

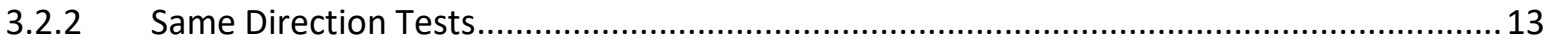

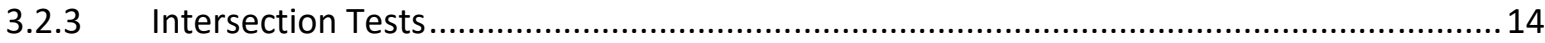

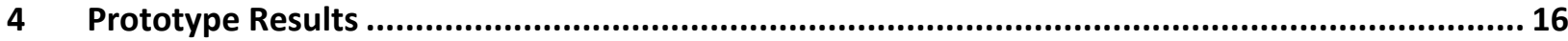

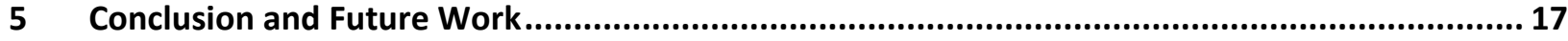

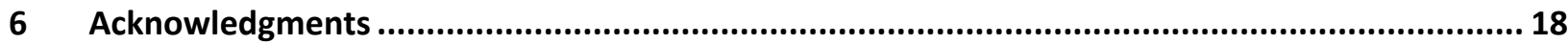

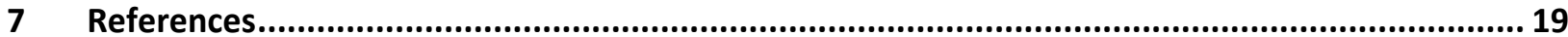




\section{List of Tables}

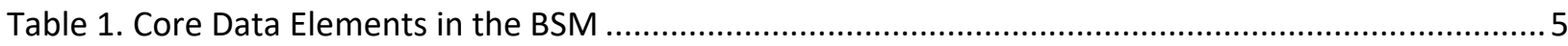

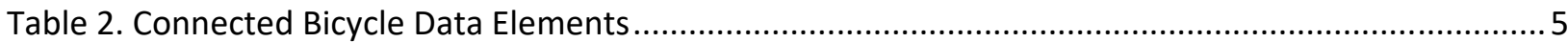

\section{List of Figures}

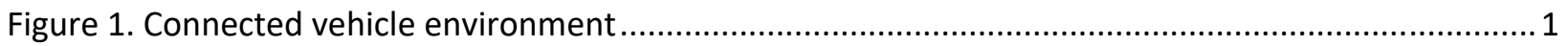

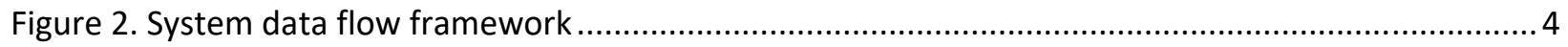

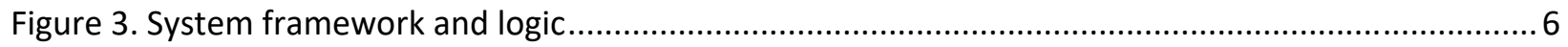

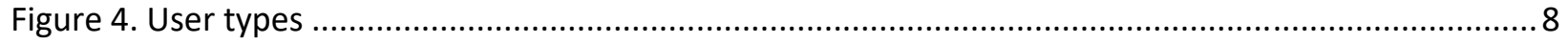

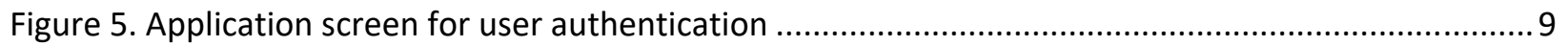

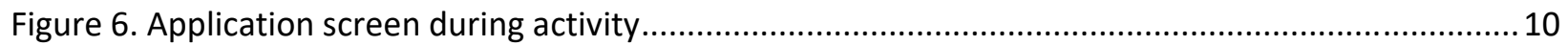

Figure 7. Application screen with warning ............................................................................................. 10

Figure 8. User movements considered for testing: a) opposite direction, b) same direction, and c) at

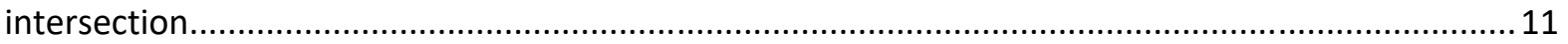

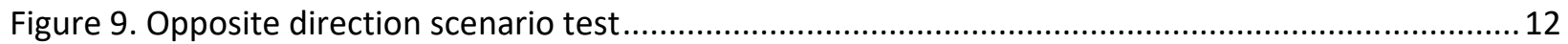

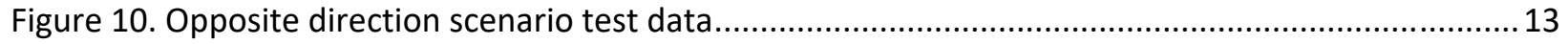

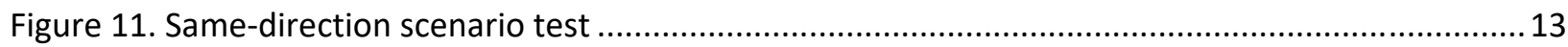

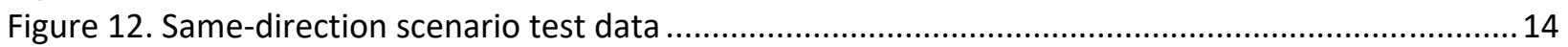

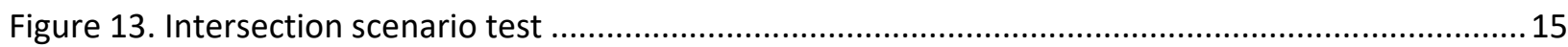

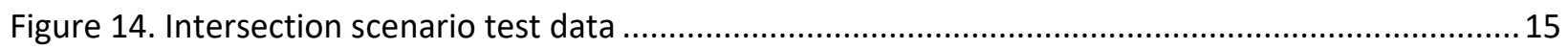




\section{I NTRODUCTION}

Due to rapid developments in the automobile industry in the past several decades, the increasing use of vehicles has caused serious safety and operation efficiency issues in terms of traffic crashes, congestion, and loss of productivity. Therefore, a sustainable multimodal transportation system has been advocated to address these traffic issues, in which bicycling, including personal cycling and bike-sharing programs, have been making a comeback as a viable and popular mode for short trips and commutes between origins/destinations and the nearest public transit (bus, metro, ferry, train, etc.) station for sustainability and economic value. However, bicyclists suffer high crash and fatality rates due to their excessive exposure to traffic compared to other modes [1]. For example, in 2016-2018, there was an annual average of 7,028 crashes involving bicyclists in Florida, resulting in an average of 139 bicyclist fatalities and 6,243 bicyclist injuries each year [2].

Connected vehicle (CV) technology has significantly advanced in recent years for traffic safety due to investments in deployments that have focused on motor vehicles (primarily cars; to a lesser extent, trucks and buses), but there has been little investment in CV research from the bicycle perspective. Smartphone-equipped users or the intelligent devices carried by drivers and bicyclists are expected to provide better information (e.g., location, speed, lane position), which could be used to send warning messages to prevent crashes [3]. In a CV world shown in Figure 1, all road users have to be connected with each other and the infrastructure to benefit from the system [4].

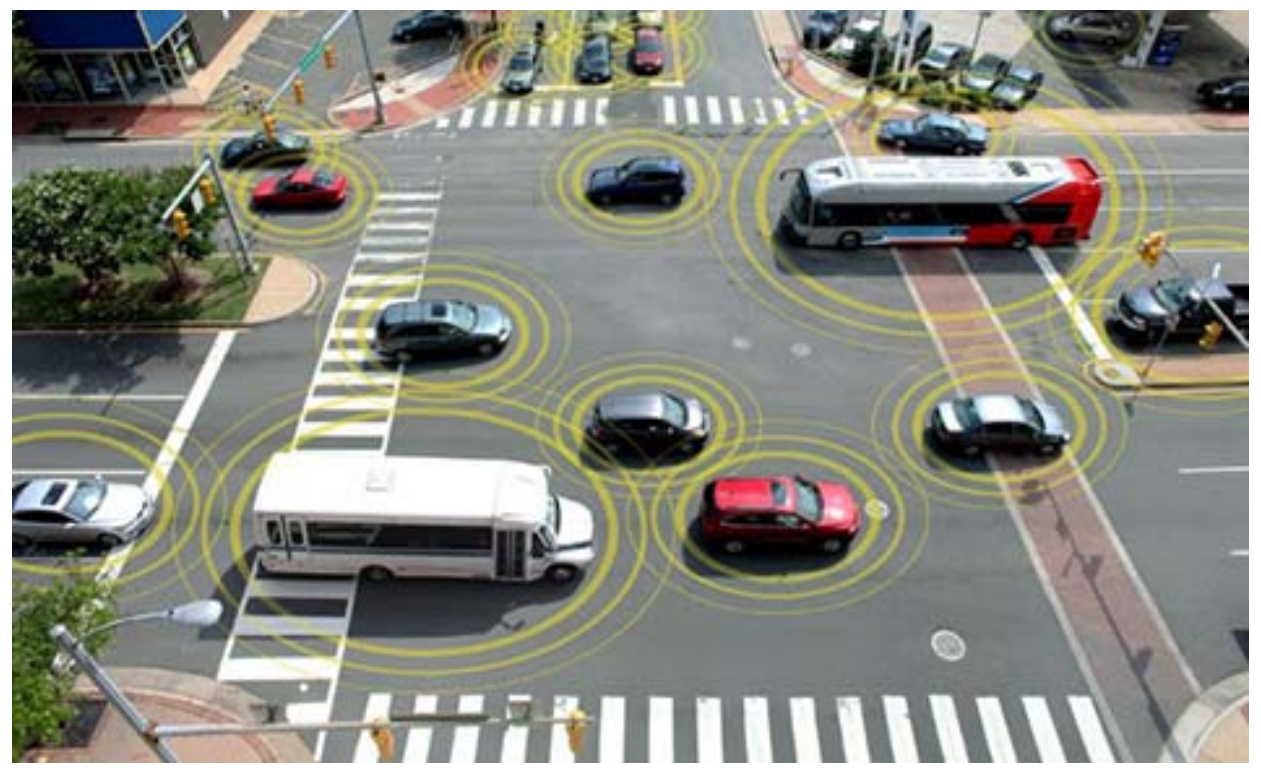

Figure 1. Connected vehicle environment

Current smartphone innovations from several peer companies take advantage of GPS and smart mobile technologies to provide a large amount of useful information regarding weather, real-time traffic, bicycle speed, positioning, route navigation, and personal cycling fitness and performance [5]. However, it is evident that these technologies provide information from a bicycle perspective but do not take into account other transportation components, including vehicles and the infrastructure, particularly traffic signals. With these advancements, it is of practical importance to develop connected bicycle techniques to improve bicyclist safety, increase the proportion of bicycle use in daily commuting, and, therefore, increase the sustainability of the entire multimodal transportation system. 
A fully-connected bicycle system should enable safe, stable, and effective wireless communications between bicycles and vehicles (B2V) and between bicycles and infrastructure (B2I). A connected bicycle will use low-cost sensors and transmitters as well as GPS, smartphone, Bluetooth, and other wireless communication systems to transmit its position, direction, speed, behavior, and other critical information to nearby vehicles, bicycles, and infrastructure to attain 360-degree awareness and to continuously receive information of interest from these parties on the road for safer and more efficient cycling. This research project proposed a pilot study to investigate the mechanism for bicycle-toeverything (B2X) communications and to develop a smartphone-based connected bicycle prototype to enhance the safety and operation efficiency of a sustainable multimodal transportation system. Connected bicycle technologies are designed to save lives, improve personal mobility and traffic operation efficiency, enhance economic productivity, reduce environmental impacts, and transform public agency operations.

Connected vehicle innovation supports every vehicle on the road communicating with other vehicles so they can share information and increase safety. If every vehicle on the road is aware of the surrounding vehicles' information, drivers can use that information to make safer driving decisions and avoid potential crashes when conflicts exist. In addition, using smartphones for this task is relatively easy and, since technological advancements are rapidly increasing their capabilities, they are the perfect tool to leverage to add all users on the increasing platform of connected vehicles.

Previous research has pioneered the notion of a bicycle as a data collection platform [6] and has inspired the B2X framework proposed. In addition, several studies have been conducted to improve bicycle safety by using in-vehicle or smartphone devices [3, 7-9]. Alert systems designed and tested have shown promise to increase safety by bicycle localization and trajectory prediction. Miah et al. [10] showed in their study that a system could provide accurate and reliability motion of bicycle using microelectromechanical systems (MEMS) sensor configurations on the bicycle and that it is possible to collect and measure various behavior factors including riding comfort using an instrumented bicycle [11]. Most studies have used either instrumented bicycles/vehicles or complex algorithms that are not feasible for implementation with standard devices used by the average person [6, 7, 12-21]. As in previous studies and similar to other CV research, surrogate measures of safety (e.g., time-to-collision [TTC], and postencroachment time [PET]) could be used to determine when a dangerous situation (conflict) between a bicycle and vehicle is imminent [12]. Therefore, a practical solution to this problem needs to be developed and tested to be widely available and be adopted by more road users.

In selecting technologies for development of the proposed system, two main communication methods were considered. First, the current communication protocol for connected vehicles used in the U.S. is the Dedicated Short Range Communication (DSRC) band. On December 17, 2003, the Federal Communications Commission (FCC) adopted a Report and Order establishing licensing and service rules for the DSRC Service in the Intelligent Transportation Systems (ITS) Radio Service in the 5.850-5.925 GHz band (5.9 GHz band) [22]. This technology uses on-board units (OBUs) and roadside units (RSUs) to send and receive the Basic Safety Message (BSM), which comprises the core of the communication between units. The OBU requires automotive power (12 volts DC) to work. Some electric bicycles might support this power, but the average bicyclist does not have access to such power without an additional battery (and its associated weight) on the bicycle. In addition, an OBU currently costs several hundred dollars, which likely is too high for the average bicyclist. The second system considered was communication via 
smartphone using Bluetooth, Wi-Fi Direct, or cellular technologies to transmit the required data between devices.

In reviewing the available communication protocols, Bluetooth and Wi-Fi Direct can provide connectivity between two devices but have high latency times and require close proximity. This made them less than ideal for use for the B2X application. Therefore, it was determined that cellular data transmission was the only feasible technology to achieve the connection and transmission of data between devices in this particular setting.

This report describes the selection, design, and testing of a smartphone application to alert bicyclists and drivers of imminent dangerous situations. The remainder of the report is structured as follows: the system design is described first, followed by the design of the prototype and test of different scenarios. The results of the prototype test are discussed, and the report concludes with findings and future work.

\subsection{Study Objectives}

The study's goal was to develop a proof-of-concept prototype and test a method to broadcast BSMs via smartphone for connected vehicles and use them to alert users of imminent crashes in established scenarios relevant to bicycles. Following are the specific objectives:

- Review existing solutions for connected/autonomous vehicles and identification of the techniques that can be useful for bicycle/vehicle interactions.

- Determine useful parameters such as speed, acceleration, latitude, longitude, etc., and system requirements including GPS, Wi-Fi, etc., for communication between bicycles and vehicles.

- Develop a mechanism for information transmission for communication between bicycles and vehicles using a smartphone-based system.

- Establish a proof-of-concept prototype for a smartphone-based connected bicycle/vehicle using selected hardware, software, and other components to include sensors, smartphone, mobile application, user interface, etc. 


\section{SYSTEM DESI GN}

Based on the literature review completed and the objectives of the project, it was determined that the existing DSRC technology was not realistic for a bicycle setup since a traditional bicycle does not have a power source for the DSRC radio required to communicate with other units. In addition, the decision between DSRC and $5 G$ technologies for communication continues to be debated, and any of them could be selected for CV communications in the future. Therefore, the system would be designed under a framework of cellular technology with the current 4G LTE bandwidth available in most metropolitan areas in the U.S. and other countries. Furthermore, for a practical system, the most straightforward application is achieved via existing technologies. An application for a smartphone could be successful in that it is the most cost effective and practical solution that can be implemented now, without the requirement of new technologies. Since most people own and use a smartphone, it is a realistic assumption that this type of system can be put in place rather quickly in the near future.

The system was designed with the framework shown in Figure 2. A user with a smartphone uses an application that collects information from the phone's sensors and transmits this information to a cloudbased server. The server collects information from all users within the network and tracks certain parameters discussed further below. Using the data collected, an algorithm calculates the time to collision (TTC) based on the spatial information between two or more users. If a threshold is reached, then the server sends notifications (warnings) to the users about an imminent collision. The objective is to provide the warning such that one or both users changes their path or speed to avoid the collision.

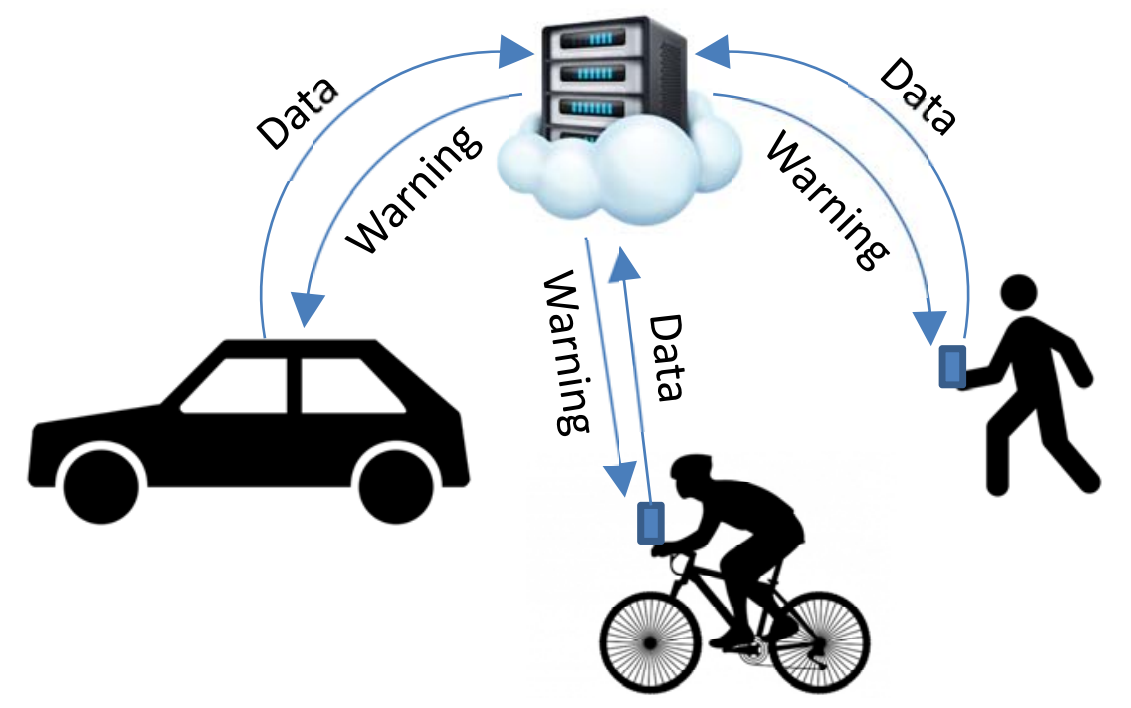

Figure 2. System data flow framework

\subsection{I dentification and Selection of Parameters}

The parameters to be used in this system had to meet two criteria: 1) provide the basic information as close to the BSM currently used and 2) be able to be collected using any basic smartphone.

The current standard for DSRC communication SAE J2735 [23] outlines the core data to be included in the BSM part 1. Table 1 shows the data elements within the BSM. Some are relevant only for motor vehicles, i.e., transmission state, steering wheel angle, and brake information. As shown in Table 1, the second criterion mentioned above was considered for implementation into the design of the system. 
Table 1. Core Data Elements in BSM

\begin{tabular}{|l|l|c|}
\hline \multicolumn{1}{|c|}{ BSM Core Data Element } & \multicolumn{1}{|c|}{ Description } & Criterion 2 Satisfied? \\
\hline msgCnt & Message count & Yes \\
\hline Id & Temporary ID & Yes \\
\hline secMark & Milliseconds within 1 minute & Yes \\
\hline Lat & Latitude & Yes \\
\hline Long & Longitude & Yes \\
\hline Elev & Elevation & Yes \\
\hline Accuracy & Positional accuracy of device & Yes \\
\hline Transmission & State of transmission of vehicle & No \\
\hline Speed & Speed of vehicle & Yes \\
\hline Heading & Heading of the vehicle & Yes \\
\hline Angle & Steering wheel angle & No \\
\hline AccelSet & Acceleration in 3 axis & Yes \\
\hline Brakes & Brake system status & No \\
\hline
\end{tabular}

Based on the parameters of the BSM and the availability to collect these using a smartphone, the decision was made to collect the information that met both criteria. Table 2 shows the parameters selected for collection into the system. In addition, the calculated parameters are shown based on data collected from the devices.

Table 2. Connected Bicycle Data Elements

\begin{tabular}{|c|c|c|c|}
\hline $\begin{array}{c}\text { Connected Bicycle } \\
\text { Data Element }\end{array}$ & Description & Units & Method \\
\hline msgCnt & Message count & & Smartphone \\
\hline Id & Id of user & & Smartphone \\
\hline User Type & Type of user (car, bike, ped) & Epoch timestamp & Smartphone \\
\hline secMark & Milliseconds & Degrees & Smartphone \\
\hline Lat & Latitude & Degrees & Smartphone \\
\hline Long & Longitude & Meters & Smartphone \\
\hline Accuracy & Positional accuracy of device & $\mathrm{m} / \mathrm{s}$ & Smartphone \\
\hline Speed & Speed of vehicle & Degrees & Smartphone \\
\hline Heading & Heading of vehicle & $\mathrm{m} / \mathrm{s}^{2}$ & Smartphone \\
\hline AccelSet & Acceleration in 3 axis & $\mathrm{m}$ & Calculated using lat, long \\
\hline Distance & Distance between 2 users & $\mathrm{m}$ & Calculated using speed, accel \\
\hline Radius & Stopping sight distance for each user & & \\
\hline
\end{tabular}

Using the parameters in Table 2, the system would be able to track all users spatially and, in turn, make determinations as to the state of the proximity to each other, thus informing drivers/bicyclists of imminent collisions.

\subsection{System Framework}

As noted, the system has to be able to collect data from each driver/bicyclist and track their movements in relation to other users in the system. The conceptual framework for the system has two main parts: 
the smartphone application or user part and the cloud-based database part where all data and calculations are performed.

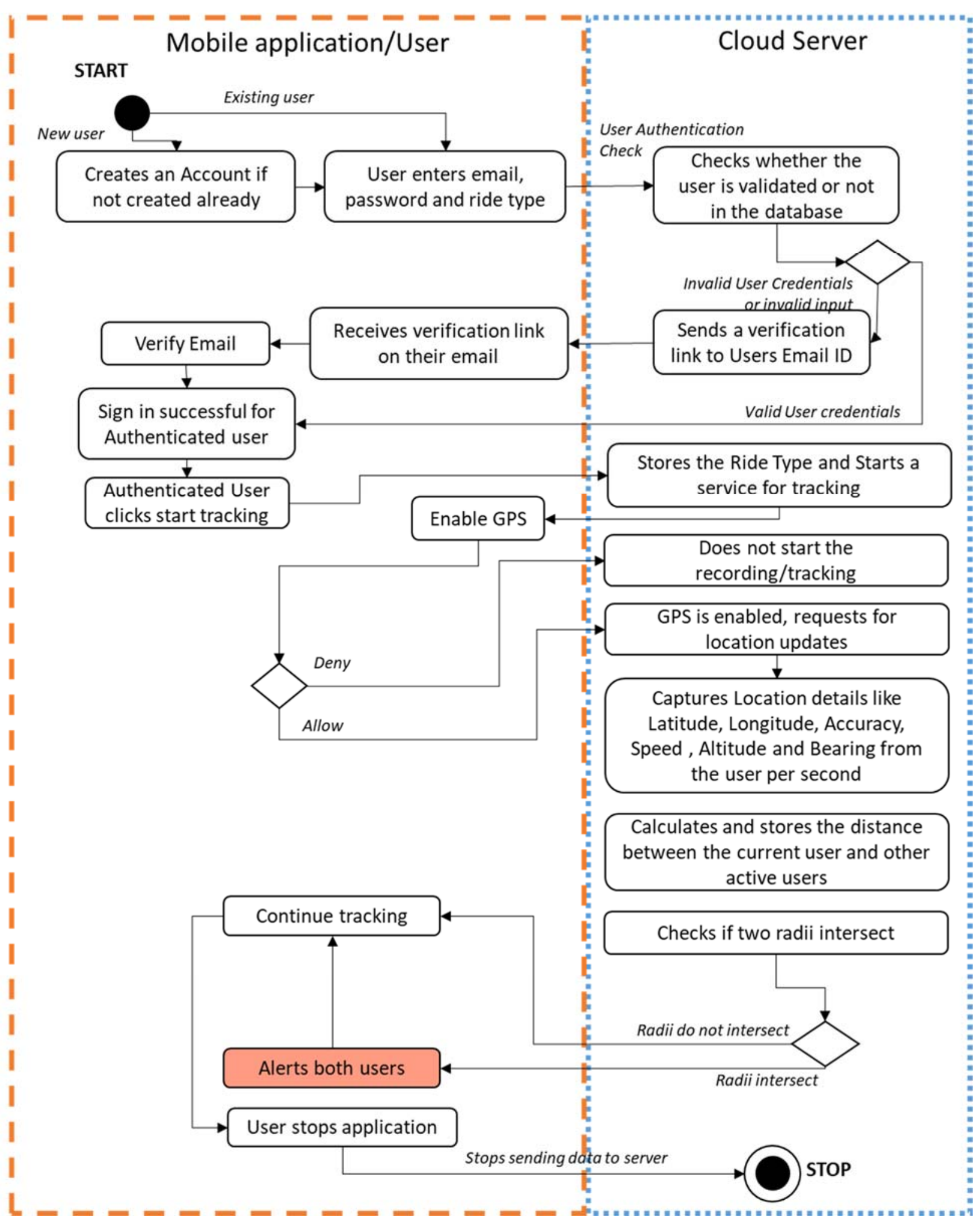

Figure 3. System framework and logic 


\section{PROTOTYPE DESI GN AND TEST}

The design of the system must include the following flow:

1. Collect information from user smartphones into a cloud server.

2. Provide a way to track user locations in relation to each other.

3. Identify conflicts using specific thresholds and deliver messages (warnings) to users.

\subsection{Prototype Design}

Using an open source application for Android OS@ developed for location data collection, the team modified the data collection protocol to include only the elements listed in Table 2. This information was transmitted with a frequency of $1 \mathrm{~Hz}$ (once per second), the highest rate a standard GPS chip used in smartphones can record at this time. The information is collected and sent in real time via cellular or WiFi connection to a cloud-based server, as shown in Figure 1. The application is available for anyone to try/test further.

\subsubsection{Data Collection and Algorithm}

The primary feature of the system is that each user is tracked anonymously via their coordinates collected by the phone and sent to the server. The system calculates the distance between each user, with any other user providing information. As the differential speed between users may be large, rather than using time to collision (TTC), the system uses the equation for Stopping Sight Distance (SSD) as defined by Eq. (1):

$$
S S D=V_{1} t_{r}+\frac{V_{1}^{2}}{2 g\left(\frac{a}{g} \pm G\right)}
$$

Where:

$$
\begin{aligned}
& V_{1}=\text { Initial vehicle speed }(\mathrm{km} / \mathrm{h}) \\
& t_{r}=\text { Driver reaction time }(\mathrm{sec}) \\
& g=\text { Gravitational constant }\left(9.81 \mathrm{~m} / \mathrm{s}^{2}\right) \\
& a=\text { Deceleration rate }\left(\mathrm{m} / \mathrm{s}^{2}\right) \\
& G=\text { Grade of the road }
\end{aligned}
$$

For traffic engineering, the reaction time is taken as $2.5 \mathrm{sec}$, with $90 \%$ of population being able to react in less than $2 \mathrm{sec}$ [24]. In addition, the road grade is equal to zero, as this is assumed for a flat road (field trial was in Florida). The inputs provided by the data collected from the phone are the instantaneous speed and acceleration (negative for deceleration). For the purposes of this study, SSD $=R$.

In addition to the calculation of radius for each user, a minimum value for this $R$ was set for each user type. These values were set to $20 \mathrm{~m}(65.6 \mathrm{ft})$ for vehicles, and $10 \mathrm{~m}(32.8 \mathrm{ft})$ for bicycles. Thus, when users are $30 \mathrm{~m} \mathrm{(100} \mathrm{ft)} \mathrm{apart,} \mathrm{the} \mathrm{minimum} \mathrm{distance} \mathrm{is} \mathrm{met} \mathrm{(Figure} \mathrm{4).} \mathrm{The} \mathrm{distance} \mathrm{between} \mathrm{the} \mathrm{two}$ points can be calculated using many methods such as the Haversine formula, the spherical law of cosines, equirectangular approximation, etc. Each coding language used has functions to achieve this 
with little difference for short distances. The system has separate elements as described in the following sections.

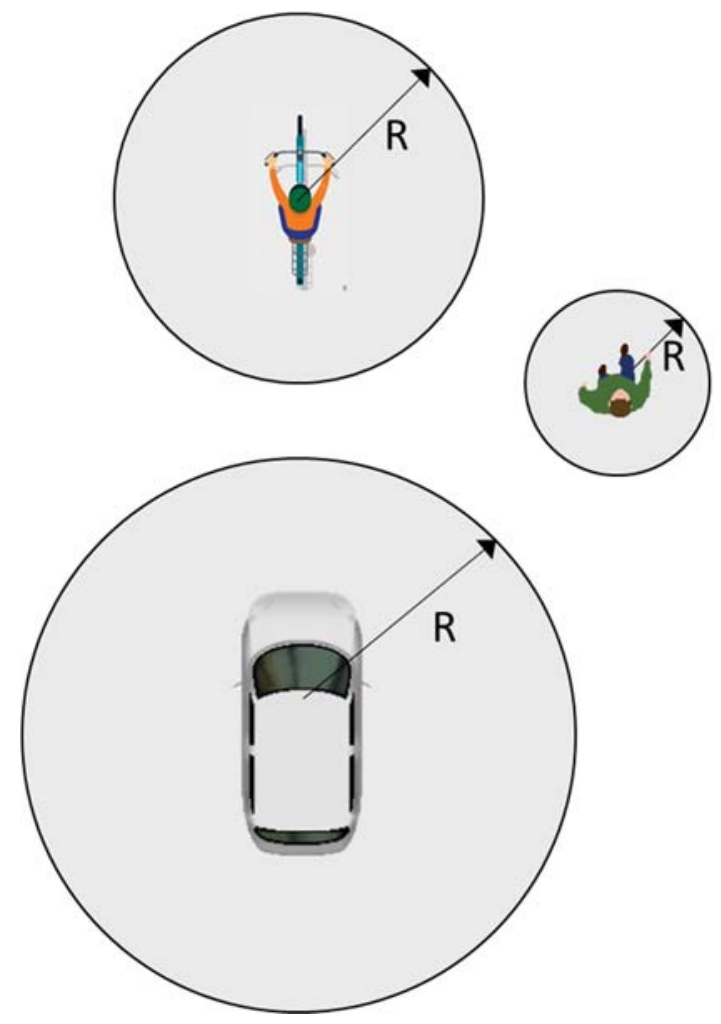

Figure 4. User types

\subsubsection{User I dentification and Authentication}

Each user installs the application on his/her smartphone and opens the application. As with any standard application, the first screen asks the user to enter his/her email address and password to create an account (Figure 5a). If a user already has an account, he/she can "sign in" instead of creating a new account. Once signed in, the application asks the user to verify the email address by sending a secured link to the email address entered. Once the user verifies the email, he/she can proceed with the use of the application features. The database assigns a unique token to each user for identification. This is how the database identifies and differentiates the users. Only authenticated users are allowed to send data to the server (Figure $5 b$ ). 


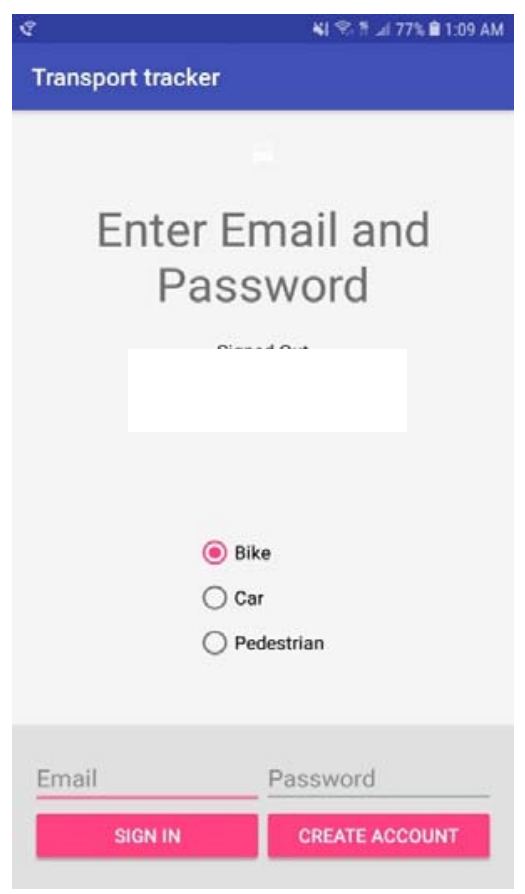

a)

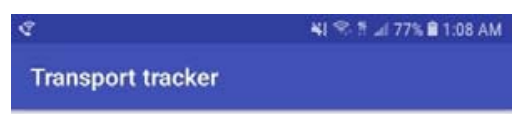

Enter Email and

Password

Email User: test@test.com (verified: false)

Firebase User: af3y8sSoc8WqrMitYfQkmmCDQwx1

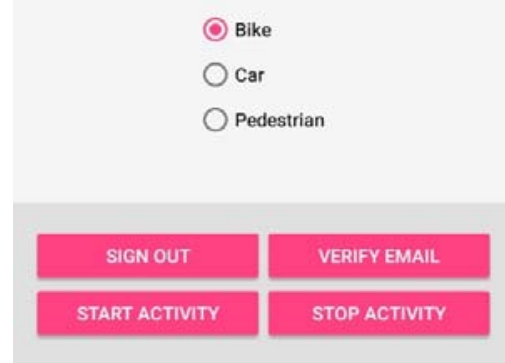

b)

Figure 5. Application screen for user authentication

\subsubsection{Activity Selection}

For the purpose of this study, the team had three users-a bicycle, a vehicle, and a pedestrian. The same user can select a different type to match the activity he/she is performing each time he/she uses the application (Figure 5). This selection also changes one main feature-the minimum radius of the circle that defines each user.

\subsubsection{Activity Recording}

After a user selects his/her type, he/she can start the activity. During the activity, the user can run other applications while this application operates in the background. The user can return to the application to stop the activity and data collection of his/her location (Figure 6). 


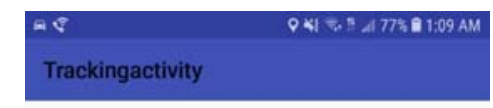

Figure 6. Application screen during activity

\subsubsection{Warning Generation}

After the user begins his/her activity and data collection commences, the system uses the algorithm described in Error! Reference source not found. to determine if two users are on a collision course and sends them a warning that varies depending on the conditions they are in at the given moment (Figure 7). The warning was presented as a notification for the prototype, but it can be changed to a pop-up window in the future.

$$
\begin{aligned}
& \text { Transport Tracker } \\
& \text { Collision Alert: You might get crash }
\end{aligned}
$$




\subsection{Prototype Test}

To test the algorithm, provide proof of concept, and collect data to fine tune parameters, a series of tests was performed with a bicyclist and vehicle both equipped with the application. Each user signed-in to the system as his/her corresponding user type and was assigned the appropriate parameters. The tests were conducted on a closed road at the University of South Florida. The tests followed the three categories of movement shown in Figure 8, movements commonly observed in real-life scenarios where a bicyclist rides in a bike lane or on the road. In each case, both users receive warnings so they pay attention and avoid a conflict.

Each scenario was tested multiple times to collect adequate data and confirm that the algorithm worked as expected. The tests shown in the figures that follow are only examples of the overall testing conducted to aid in describing the scenarios.

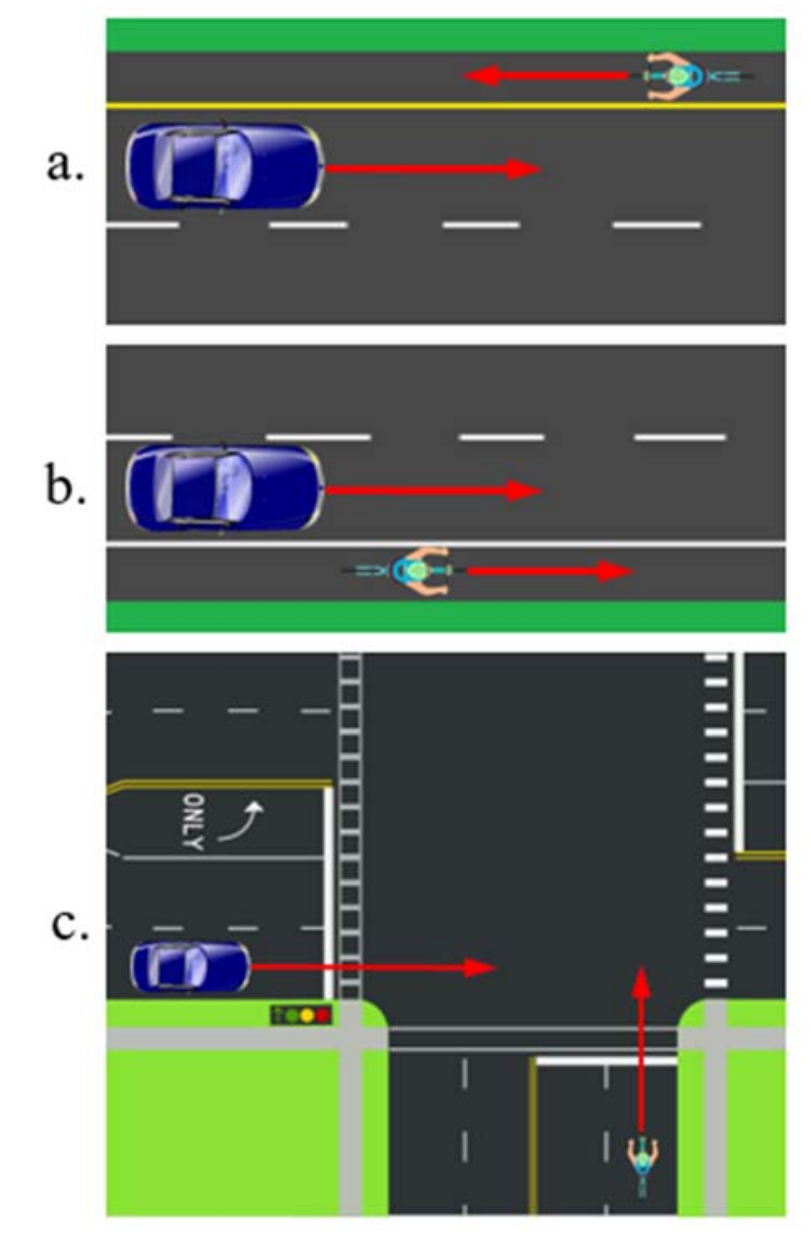

Figure 8. User movements considered for testing: a) opposite direction, b) same direction, and c) at intersection

\subsubsection{Opposite Direction Tests}

The opposite direction scenario test was conducted for two users (a vehicle and a bicycle) moving towards each other. Figure 10 shows an example conducted under this scenario. The bicycle was moving southwest and the vehicle northeast. In Figure 10, the markers show the respective user position as 
recorded by the GPS of his/her smartphone and is color-coded for normal condition where the user is traveling without warnings and for warning condition under which the user is receiving warning on his/her smartphone. The warnings were delivered while the parameters were met, i.e., the user received warnings continuously as long as he/she was under "warning conditions."

In the test shown in Figure 10, the bicyclist received two warnings and the vehicle received three. Also as shown, the vehicle received the warning before it reached the bicycle, at a distance of approximately $30 \mathrm{~m}(100 \mathrm{ft})$. The vehicle was traveling at $30 \mathrm{mph}$ and the bicycle at $7 \mathrm{mph}$. Of note is the actual location of the markers with respect to the satellite image. As shown on the two maps, the markers are not as accurately portrayed because of GPS inaccuracy and mapping the location on the basemap. The most important variable is the spatial relationship between the two users in space and time since the algorithm has no map representation or consideration.

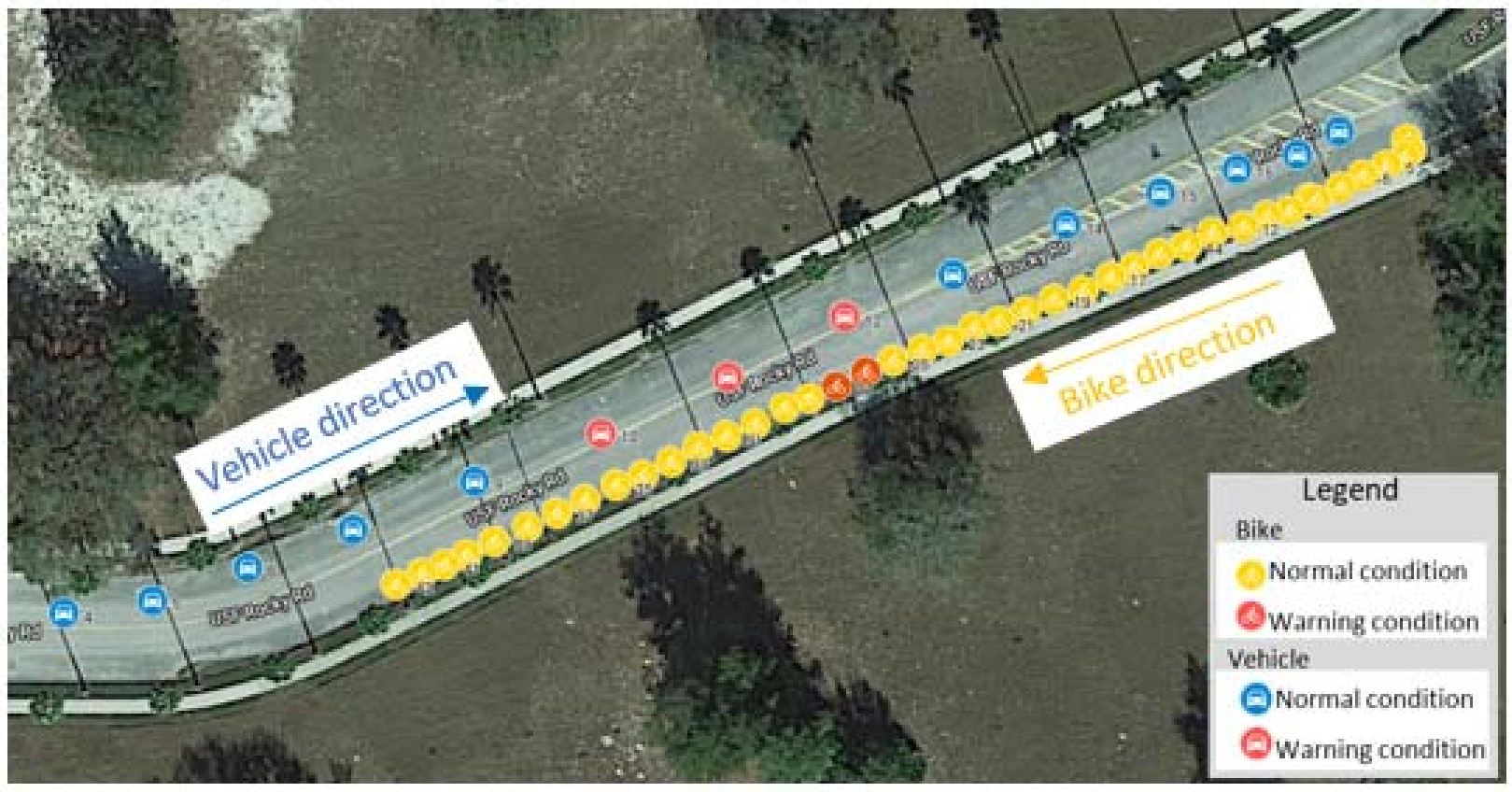

Figure 9. Opposite direction scenario test

Figure 10 shows the distance in meters between the vehicle and bicycle, the time to collision (TTC), and the moments in which a warning was displayed for the users. The $x$-axis shows elapsed time in seconds. For this example, the warning is displayed for three seconds for the vehicle (during seconds 5,6 , and 7 on the $x$-axis). The warning was displayed for two seconds for the bicyclist as shown in Figure 10.

The plot also shows that the distance between the vehicle and bicycle at the second before the warning was issued was 30 meters ( $100 \mathrm{ft}$ ). This was due to the parameter described earlier-the minimum radius for bicycle was 10 meters ( $\sim 33 \mathrm{ft}$ ) and the minimum radius for the vehicle was 20 meters ( $66 \mathrm{ft}$ ). When the distance between the two users dropped below $30 \mathrm{~m}(10 \mathrm{~m}+20 \mathrm{~m})$, then the warning was issued and continued to be displayed until the distance increased more than 30 meters. 


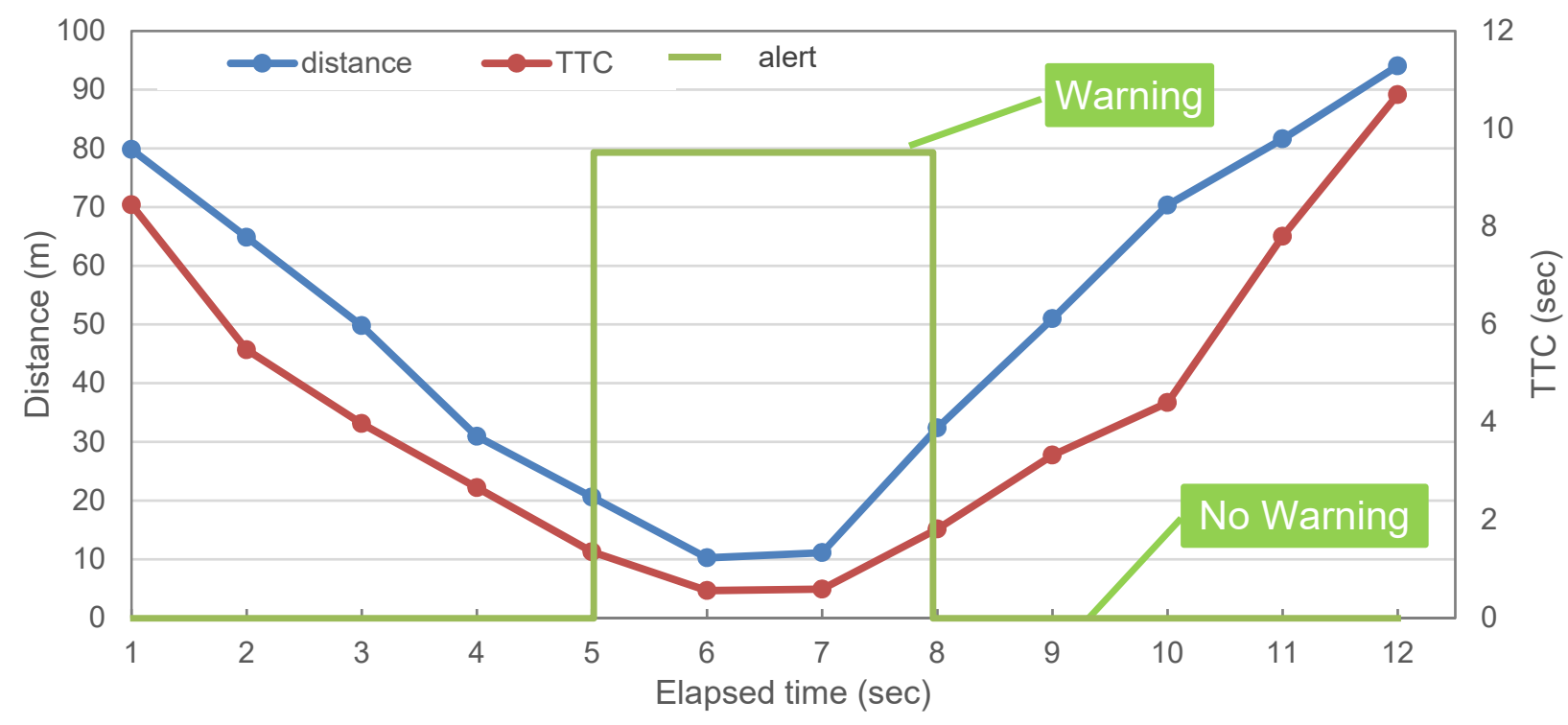

Figure 10. Opposite direction scenario test data

\subsubsection{Same Direction Tests}

The second type of movement is the most common scenario, in which a bicyclist and a vehicle are traveling in the same direction. In this case, the bicycle is traveling in the bike lane or travel lane and the vehicle is approaching the bicycle from the rear at a higher speed. Under normal conditions, the vehicle passes the bicycle without conflict, but sometimes, depending on the width of the travel lane or debris in the bike lane, the bicycle and vehicle come to a collision course. In this case, it is useful to warn both users so they pay attention to each other. Figure 11 shows an example of a same-direction scenario test. The vehicle is traveling at $25 \mathrm{mph}$ and the bicycle at $8 \mathrm{mph}$ with a distance of $106 \mathrm{ft}$ between them before the warning was sent.

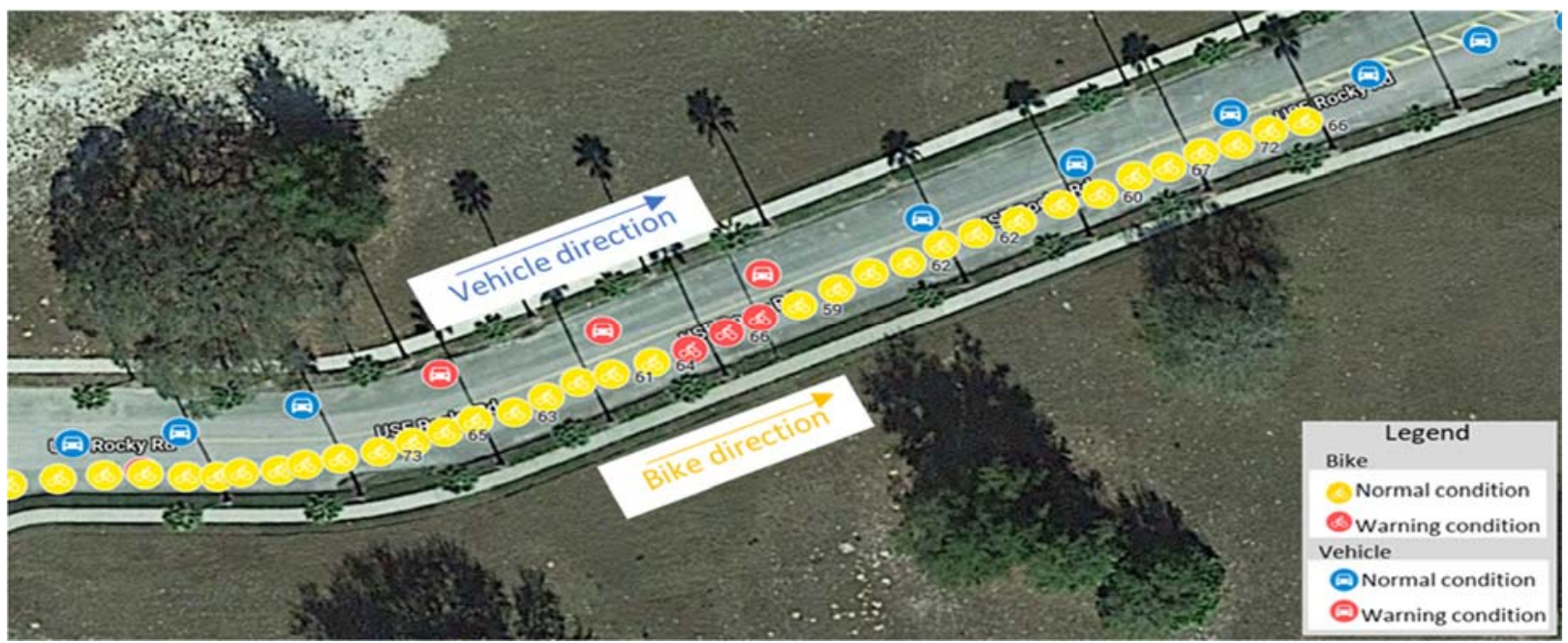

Figure 11. Same-direction scenario test

Figure 12 shows the distance, TTC, and vehicle warning for the same test scenario. Similar to the opposite direction scenario, the vehicle approached the bicycle and received three warnings after the 
distance between the two users dropped below $30 \mathrm{~m}$. In this case, the warning stopped being generated as soon as the distance between the vehicle and bicycle started to increase, since this signals that the two users are not approaching each other anymore but rather are separating (no more danger).

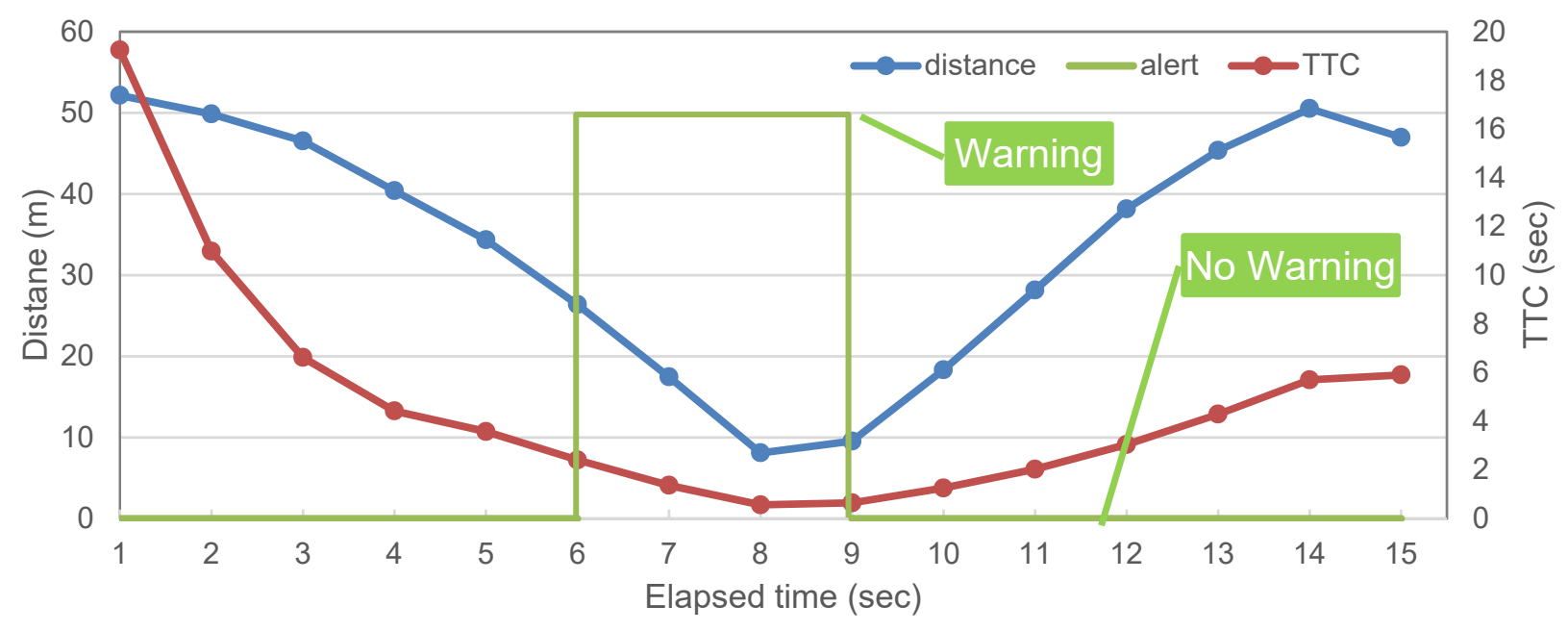

Figure 12. Same-direction scenario test data

\subsubsection{Intersection Tests}

The third scenario is also common between vehicles and bicyclists, in which the two users come to an intersection and will cross paths. The intersection control is not important, as this will work at any type (stop sign, roundabout, traffic signal or none). During this scenario, the vehicle and bicycle are approaching each other on collision course, and the warnings are issued when the parameters are met (i.e., distance between users) falls below a certain threshold. Figure 13 shows a test ion which the vehicle is on a side street coming to a stop-controlled intersection, and the bicycle is riding on the sidewalk and crosses on the crosswalk. The warning is presented to both the vehicle and bicycle before they reach the intersection, thereby alerting them to each other's presence.

In this case, the vehicle comes to a complete stop at the intersection and the bicycle crosses in front of the vehicle. The particular test site has a blind spot generated by shrubs in the median on the side street, and the vehicle cannot see the left side of the crosswalk. This was a perfect demonstration of the need for a warning before reaching an intersection since the driver could pull out, pass the stop bar, and move into the crosswalk to be able to see oncoming traffic from the left, thereby on a collision course with the crossing bicycle. Figure 14 shows the data collected during the intersections scenario, in which the vehicle driver was alerted for five seconds, which was the time it took the bicyclist to cross the crosswalk at the intersection. 


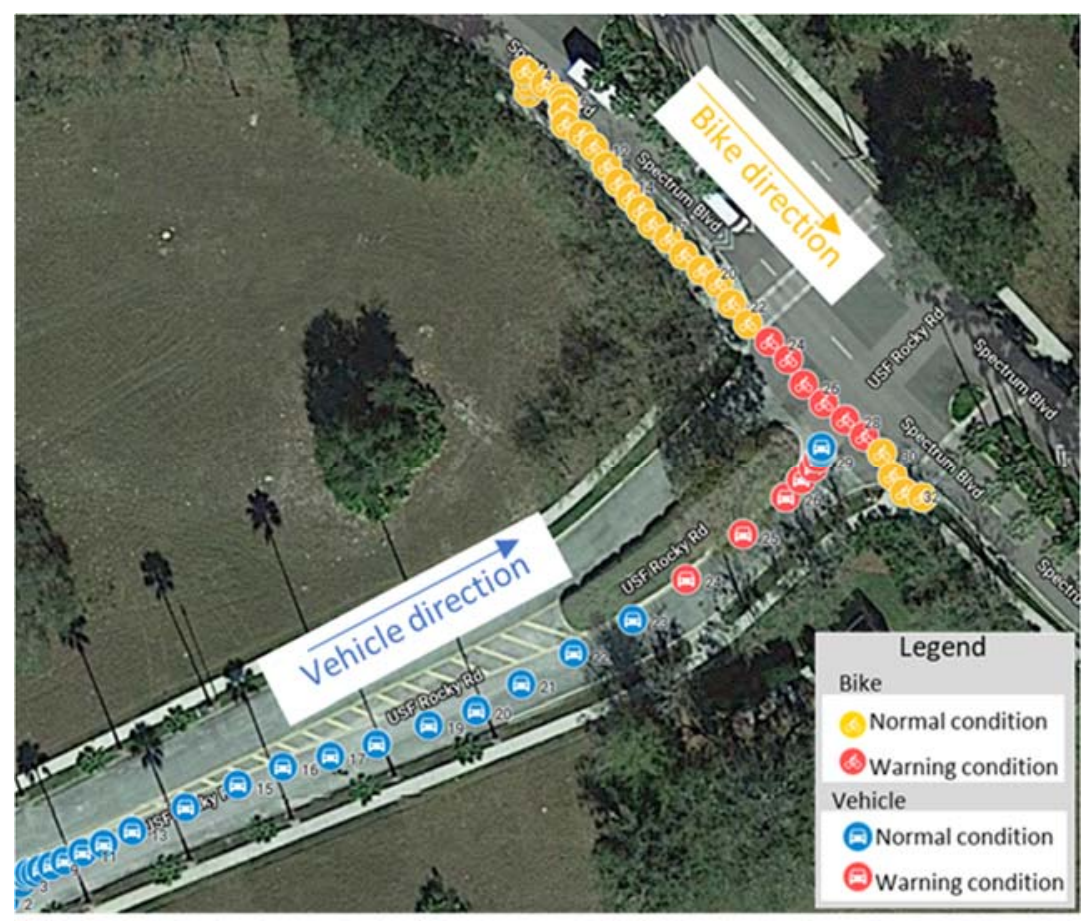

Figure 13. Intersection scenario test

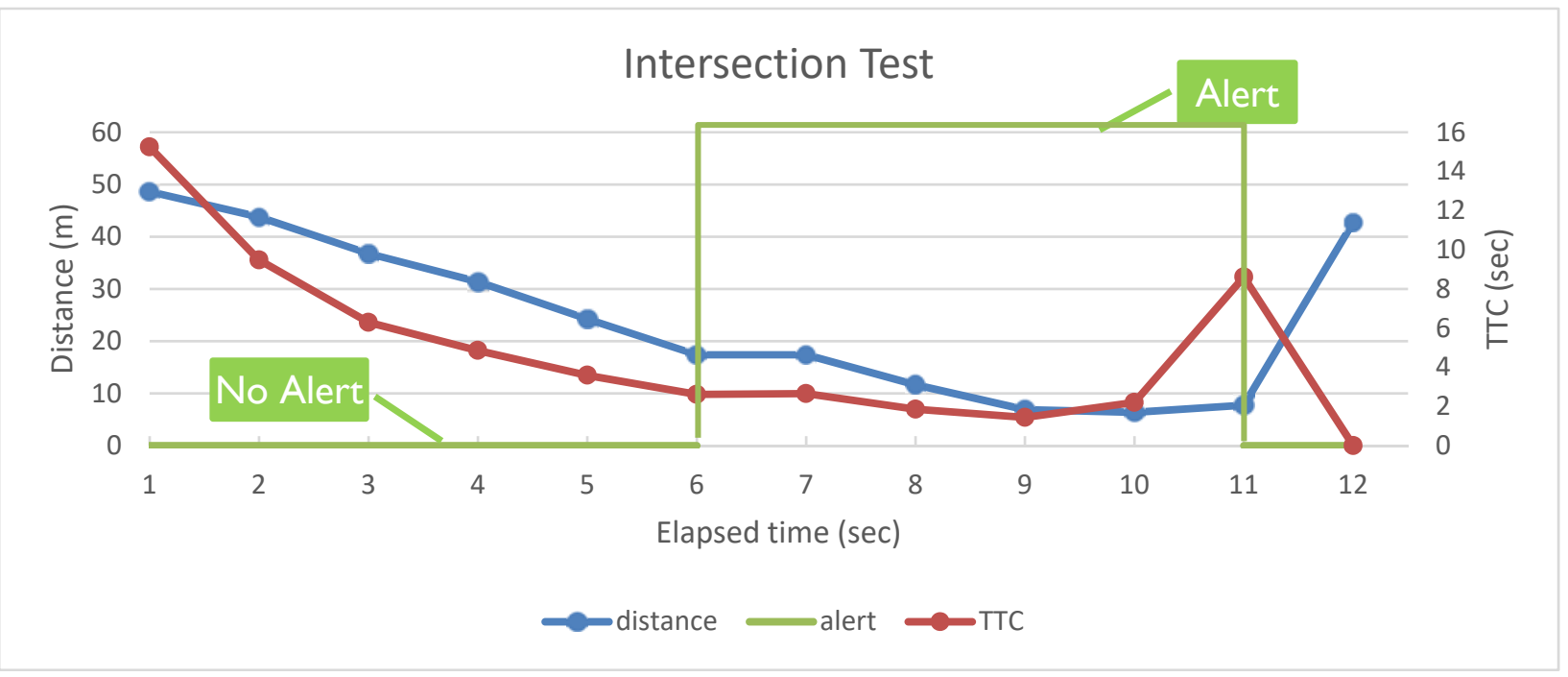

Figure 14. Intersection scenario test data 


\section{PROTOTYPE RESULTS}

The testing of the application showed promising results for the three test scenarios considered. The three test scenarios (opposite direction, same direction, intersection) cover the majority of cases in which a vehicle and a bicycle interact, especially when the bicyclist is using bike lanes or travel lanes. The testing of the application showed that for all three cases, the algorithm was able to use the collected data from the smartphones to establish their proximity to each other and alert the users when the distance between them dropped below a certain threshold. As shown in the data collected and during experimentation, the algorithm was able to deliver the first warning when the users were approximately $30 \mathrm{~m}$ (100 ft) from each other. This was the set parameter for the tests, in which the vehicle had a minimum radius of $20 \mathrm{~m}$ and the bicycle had a minimum radius of $10 \mathrm{~m}$. These parameters are configurable and can be changed to accommodate shorter or longer distances between users. The only case in which the $30 \mathrm{~m}$ distance was not met was the intersection test. As shown in Figure 14, the users were approximately $24 \mathrm{~m}$ from each other before the warning was issued.

The application takes into consideration the users' heading (bearing) to establish the direction under which they are approaching each other. This way, a custom message for each warning is delivered depending on the scenario and user. In addition, the tests showed a few limitations of the current system and opportunities for improvement. One is that the accuracy of a single GPS point cannot be trusted for accuracy due to GPS drifting (coordinates are not accurate for a single point among many that are) and, therefore, the calculations cannot be guaranteed to work if using single GPS points. Coupled with the fact that the frequency of points is only $1 \mathrm{~Hz}$ (1 per second), this can result in erroneous estimations, especially when two users are close to each other. However, this can be improved in two ways: first, the algorithm can be changed to consider the moving average of a few points (instead of one), thereby reducing the error if a single point happens to be off, and second, the algorithm can fill in the gaps (interpolate) between points to an increased frequency close to $10 \mathrm{~Hz}$ (10 points per second), which is the current standard for connected vehicles using DSRC technology. 


\section{CONCLUSION AND FUTURE WORK}

The proposed solution for connected bicycles offers a platform and framework to connect bicycles and vehicles equipped with just a smartphone for $\mathrm{V} 2 \mathrm{X}$ applications for safety. The proposed algorithm has the potential to warn both drivers and bicyclists of imminent dangerous situations that occur when they two types of users come in close proximity to each other. The algorithm works anywhere as long as the two users use the developed application to provide basic location data during their trips. The system was designed to be used using current cellular communication such as 4G LTE but can be greatly improved in the future with the use of $5 \mathrm{G}$ communication technology.

During testing, three different scenarios were considered-users traveling in the same direction, users traveling in opposite direction, and users traveling at (or close to) perpendicular direction. These three scenarios represent the majority of conflict types between vehicles and bicycles on the road. The application was developed for smartphones using the Android ${ }^{\circledR}$ operational system but can be expanded to include Apple' ${ }^{\circledast}$ iOS. During testing for all three scenarios, the algorithm was able to send warnings to the two users meeting the parameters set for each case. The system was built on a cloud-based platform, thereby allowing for scaling up when more users are added. To avoid issues in handling large number of users simultaneously, the system can be improved by using a grid network, where calculations for user in proximity are made for a small area, thus keeping the detection of users localized.

Several limitations and improvements have been identified that can be addressed in future research:

- The accuracy of GPS can be greatly improved using the new dual frequency (L1/L5) GPS chips that can bring the accuracy to 1 meter ( $3 \mathrm{ft}$ ) [25] or using fusion algorithm to enhance accuracy of the location of the bicycle using additional sensors [26].

- The low data frequency (1 per sec) can be improved by interpolating points and filling in the gaps.

- The shape of the user boundary can be improved by changing it to an ellipse rather than a circle, thereby reducing the times when an warning is provided but not needed (false positive) but keeping true events (accurate warning) at a maximum.

These improvements can be made and tested in subsequent work on the system. 


\section{ACKNOWLEDGMENTS}

This work was supported by the National Center for Transit Research, a program of the Center for Urban Transportation Research at the University of South Florida and funded by the U.S. Department of Transportation. 


\section{REFERENCES}

1. Vanparijs, J., et al., Exposure Measurement in Bicycle Safety Analysis: A Review of the Literature. Accident Analysis \& Prevention, 2015. 84: p. 9-19.

2. Florida Crash Dashboard. 2019 [cited 2019 July 22]; Available from: https://www.flhsmv.gov/traffic-crash-reports/crash-dashboard/.

3. Greene, D., et al., An Efficient Computational Architecture for a Collision Early-Warning System for Vehicles, Pedestrians, and Bicyclists. IEEE Transactions on Intelligent Transportation Systems, 2011. 12(4): p. 942-953.

4. Connected Vehicle Basics. 2019 [cited 2019 July 22]; Available from: https://www.its.dot.gov/cv basics/index.htm.

5. Strava. 2019 [cited 2019 July 22]; Available from: https://www.strava.com/features.

6. Bigazzi, A.Y. and M.A. Figliozzi. The Portland ACE: a Portable, Low-cost, and Networds Device for Assessing Cyclists' Exposure to Air Pollution. in Ninth International Conference on Networked Sensing Systems (INSS). 2013.

7. Jeon, W. and R. Rajamani, Rear Vehicle Tracking on a Bicycle Using Active Sensor Orientation Control. IEEE Transactions on Intelligent Transportation Systems, 2018. 19(8): p. 2638-2649.

8. Liebner, M., F. Klanner, and C. Stiller. Active Safety for Vulnerable Road Users based on Smartphone Position Data. in IEEE Intelligent Vehicles Symposium. 2013. Gold Coast, Australia.

9. Gustafsson, P., et al. BikeCOM: cooperative safety application supporting cyclists and drivers at intersections. in 3rd International Conference on Driver Distraction and Inattention. 2013. Gothenburg, Sweden.

10. Miah, S., I. Kaparias, and P. Liatsis, Cyclist $360^{\circ}$ Alert: development and testing of a prototype instrumented bicycle model for the prevention of cyclist accidents, in 47th Annual Conference of the Universities’ Transport Study Group. 2015: United Kingdom.

11. Miah, S., et al., Measuring cycle riding comfort in Southampton using an instrumented bicycle, in 6th International Conference on Models and Technologies for Intelligent Transportation Systems. 2019.

12. Wu, Y., et al., Developing a Crash Warning System for the Bike Lane Area at Intersections with Connected Vehicle Technology. Transportation Research Record, 2019. 2673(4): p. 47-58.

13. Feng, F., S. Bao, and M. Delp. Vehicle Lane Encroachment When Drivers Overtaking Bicyclists - An Examination Using Naturalistic Driving Data. 2018.

14. Fraser, M., L. Meuleners, and K. Chow. Reckless cyclists or impatient drivers? A naturalistic study of group riding in Perth, Western Australia. 2018.

15. Haus, S.H. and H.C. Gabler. Characteristics of Vehicle-Bicycle Crashes and Near Crashes Using Naturalistic Driving Data. 2018.

16. Kovaceva, J., et al., Drivers Overtaking Cyclists in the Real-World: Evidence from a Naturalistic Driving Study. Safety Science, 2018.

17. Lin, P.-S., et al., Naturalistic Bicycling Behavior Pilot Study. 2017. p. 104p.

18. Mackenzie, J., J. Thompson, and J. Dutschke. Development of a Device Suitable for Naturalistic Studies of Passing Distances Between Cyclists and Vehicles. 2017. 
19. Schleinitz, K., et al., The German Naturalistic Cycling Study - Comparing Cycling Speed of Riders of Different E-Bikes and Conventional Bicycles. Safety Science, 2017. 92: p. pp 290-297.

20. Bigazzi, A.Y., et al., Breath Biomarkers to Measure Uptake of Volatile Organic Compounds by Bicyclists. Environmental Science \& Technology, 2016. 50(10): p. 53575363.

21. Fernandez, M.D.a.A., Understanding Bicycle Dynamics and Cyclist Behavior From Naturalistic Field Data. IEEE Transactions on Intelligent Transportation Systems 2012. 15(1): p. 376-384.

22. Dedicated Short Range Communications (DSRC) Service. 2019 [cited 2019 July 22]; Available from: https://www.fcc.gov/wireless/bureau-divisions/mobilitydivision/dedicated-short-range-communications-dsrc-service.

23. Dedicated Short Range Communications (DSRC) Message Set Dictionary J2735_201603. 2016: SAE International.

24. Layton, R. and K. Dixon, Stopping Sight Distance. 2012, The Kiewit Center for Infrastructure and Transportation. p. 28.

25. Van Diggelen, F., R. Want, and W. Wang How to achieve 1-meter accuracy in Android. GPS World, 2018.

26. Shahjahan Miah , E.M., Ioannis Kaparias, and Nicholas Karcanias, An Innovative MultiSensor Fusion Algorithm to Enhance Positioning Accuracy of an Instrumented Bicycle. IEEE Transactions on Intelligent Transportation Systems, 2019. 